\title{
The Genotypic and Phenotypic Basis of Chickpea (Cicer arietinum L.) Cultivars for Irrigation-Based Production in Ethiopia
}

\author{
Nigusie Girma ${ }^{1}$, Asnake Fikre $^{2} \&$ Chris O. Ojiewo ${ }^{2}$ \\ ${ }^{1}$ Debre zeit Agricultural Research Center, Debre Zeit, Ethiopia \\ ${ }^{2}$ International Crop Research Institute for the Semi-Arid Tropics, Addis Ababa, Ethiopia \\ Correspondence: Chris O. Ojiewo, International Crop Research Institute for the Semi-Arid Tropics, Addis Ababa, \\ Ethiopia. E-mail: c.ojiewo@cgiar.org
}

Received: March 15, 2017

Accepted: June 15, 2017

Online Published: July 15, 2017

doi:10.5539/jas.v9n8p229

URL: https://doi.org/10.5539/jas.v9n8p229

\begin{abstract}
Development of irrigation-based chickpea production is considered the most important alternative approach in combating climate change and maximizing productivity, especially in moisture-stress areas and in areas where water and land for irrigation is available. In central Ethiopia, where production of chickpea (especially Kabuli type) is becoming an important part of agriculture, although many superior varieties (both desi and Kabuli types) are available, they have been evaluated and released based on rainfed production. Hence, there is an urgent need for evaluation of varieties suited for irrigation-based production. Towards this goal, during the 2012/13 growing season, 14 Kabuli genotypes (previously introduced) and 24 desi genotypes (nurseries obtained from ICRISAT) were evaluated independently at three and one locations respectively (Kabuli at Debre Zeit, Ambo and Werer; $d e s i$ at Debre Zeit) for production adaptation under irrigation. The parameters evaluated were date of maturity, 100 -seed weight and yield. Overall, while most Kabuli genotypes showed high adaptability to irrigation-based production at all locations, four Kabuli genotypes (X96TH-52-14/2000 $=106.7 \mathrm{DAS}$, FLIP-02-39C $=107 \mathrm{DAS}$, X98TH-51-1-3 = 107.9DAS and ICCV-07313 = 107DAS) were found to be earlier in maturity; two genotypes (ICCV-07313 $=42.5 \mathrm{~g}$ and ICCV-04305 $=37.8 \mathrm{~g}$ ) were identified as having high 100-seed weight and one genotype (ICCV-05309 $=3228.8 \mathrm{~kg} / \mathrm{ha}$ or 32 quintals $/ \mathrm{ha}$ ) out yielded all genotypes across locations. The result of combined analysis indicated five promising genotypes showing more than $20 \mathrm{~kg} / \mathrm{ha}$ yield on average. All desi varieties showed maturity dates of under four months; six genotypes showed higher 100-seed weight and eight genotypes showed promising yield responses $(>2000 \mathrm{~kg} / \mathrm{ha}$ ). From these preliminary results, it can be deduced that irrigation can play a significantly complementary role to the rainfed system, provided the genetics by management is optimized through research and innovation.
\end{abstract}

Keywords: chickpea, combined, genotypes, irrigation, rainfed, yield

\section{Introduction}

Chickpea (Cicer arietinum) is one of the most important cool-season annual grain legumes grown in more than 50 countries (89.7\% area in Asia, $4.3 \%$ in Africa, $2.6 \%$ in Oceania, $2.9 \%$ in the Americas and $0.4 \%$ in Europe) with India taking the largest share (64\%) of the global production. Countries such as Pakistan, Turkey, Iran, Australia, Ethiopia, Canada, Mexico and Iraq are also important chickpea-producing countries (Gaur et al., 2012). In Ethiopia, chickpea holds third place as a grain legume both by area coverage and volume of production, next to faba bean and haricot bean (CSA, 2016). There are two distinct types of chickpea identified (Muehlbauer et al., 1982; Jukanti et al., 2012):

Desi chickpea: It has a thick, colored seed coat. The common seed colors include various shades and combinations of brown, yellow, green and black. The seeds are generally small and angular with a rough surface. The flowers are generally pink; however, some desi types have white flowers.

Kabuli chickpea: The Kabuli type chickpeas are characterized by white seeds with ram's head shape, smooth surface and thin seed coat. The flowers are generally white. As compared to desi types, the Kabuli types have higher levels of sucrose and lower levels of fiber. The Kabuli types generally have large seeds and receive higher market price than desi types; the price value in Kabuli type generally increases as the seed size increases. 
Chickpea is cultivated for different values it provides, whether in nutrition, food and environmental rehabilitation, or in cash generation. Its nutritional value, medicinal value and market value, as compared to other annual crops, are some of the most important merits for which the crop is grown in Ethiopia. Nutritionally, the chickpea seed has $38-59 \%$ carbohydrate, $3 \%$ fiber, $4.8-5.5 \%$ oil, $3 \%$ ash, $0.2 \%$ calcium and $0.3 \%$ phosphorus. Digestibility varies from $76-78 \%$ for its protein and $57-60 \%$ for its carbohydrate (Hulse, 1991; Kumar et al., 2016). Pulses such as chickpeas are important sources of macronutrients, containing almost twice the amount of protein compared to cereal grains (Brick et al., 2003). They are good sources of fiber, folic acid, manganese, iron, magnesium, copper and zinc (Brick et al., 2003).

Medicinally, a cooked chickpea-milk (4:1) mixture has been found beneficial for infants, effectively controlling diarrhea (Jukanti et al., 2012). In addition, chickpea has been reported as an important means in controlling bronchitis, cholera and constipation; acids in chickpea seed are supposed to lower blood cholesterol levels (Jukanti et al., 2012). Regular consumption of pulses such as chickpeas prevents diabetes and reduces risk of heart disease (Jukanti et al., 2012).

The local consumption of chickpea in different forms for household use and local market has increased with time (CSA, 2015). Virtually all domestic pulse crop production is marketed through processors for export but the majority is sold on the local market and consumed locally. However, the quantity and quality of the product produced limit the ability of domestic producers to influence world markets and to consistently produce sufficient quantities to be a reliable supplier for large users.

Though most of the production of the crop in Ethiopia is restricted to rainfed fields, chickpea is assumed to be the most adaptive to drought, and responds better to small irrigation than other legume crops. If it is irrigated at the right time with the required amount of water, the yielding potential of the crop could be increased. Egypt and Sudan produce chickpea solely using irrigation and Egypt takes the first place in productivity with about 20 quintal/ha. Egypt irrigates the crop 1-2 times during the early stage of crop emergence and during flowering and maturity, while in Sudan chickpea is irrigated 5-7 times. Apart from the availability of irrigation water and land, availability of the most responsive varieties of chickpea suited to irrigation is very important. In Ethiopia, the potential suitable land and water resources for irrigation-based chickpea production surpasses many thousand hectares. Accordingly, the demand for varieties suitable for irrigation is soaring. To address this and beyond, the National Chickpea Improvement Program has put in place over location response assessment of pipeline germplasm to evaluate and characterize their inherent responses, and its stability. Therefore, this study is designed to develop cultivars suited for an irrigation-based production system in Ethiopia.

\section{Materials and Methods}

\subsection{Description of Study Area}

Experiments were conducted at Debre Zeit Agricultural Research Center, Werer Agricultural Research Center and Ambo Plant Protection Research Center at the Ethiopian Institute of Agricultural Research during 2013-2014. The agro-climatic conditions, altitude and soil characteristics of the study areas are presented in Table 1 below.

Table 1. Agro-climatic conditions, altitude and soil characteristics of the study areas

\begin{tabular}{|c|c|c|c|c|c|c|c|}
\hline \multirow{2}{*}{ No. } & \multirow{2}{*}{ Center } & \multirow{2}{*}{$\begin{array}{l}\text { Altitude } \\
\text { (m.a.s.1.) }\end{array}$} & \multirow{2}{*}{$\begin{array}{l}\text { Average Annual } \\
\text { rainfall }(\mathrm{mm})\end{array}$} & \multicolumn{2}{|c|}{ Temperature $\left({ }^{\circ} \mathrm{C}\right)$} & \multirow{2}{*}{ Soil type } & \multirow{2}{*}{ PH } \\
\hline & & & & $\operatorname{Min}\left({ }^{\circ} \mathrm{C}\right)$ & $\operatorname{Max}\left({ }^{\circ} \mathrm{C}\right)$ & & \\
\hline 1 & Debre Zeit & 1900 & 851 & 8.9 & 28.3 & Vertisols & 7.45 \\
\hline 2 & Werer & 740 & 533 & 15.2 & 32.5 & Fluvisols & $7.5-8.5$ \\
\hline 3 & Ambo & 2175 & 1260.90 & 10.02 & 26.89 & Pellic Vertisols & 6.78 \\
\hline
\end{tabular}

\subsection{Treatments}

A total of 14 early Kabuli chickpea lines were promoted from preliminary variety trial (PVT) and 24 desi chickpea lines introduced from ICRISAT were grown on-station at Debre Zeit, Ambo and Werer Agricultural Research Centers using irrigation. Planting was in four rows of plot of $4 \mathrm{~m}$ long and $1.2 \mathrm{~m}$ wide in Randomized Complete Block Design (RCBD) with three replications as shown in Figure 1. Planting was done on 10/01/2012 (for Kabuli) and 05/01/2012 (for desi) at Debre Zeit, 06/01/2012 at Ambo and 24/12/2012 at Werer since rainfall at all stations terminate before planting time and there is a dry spell following it. At all locations, the trials were surface irrigated four times using furrow irrigation and selection was made on the basis of date of maturity, 100 -seed weight and yield; analysis was done using SAS software. 

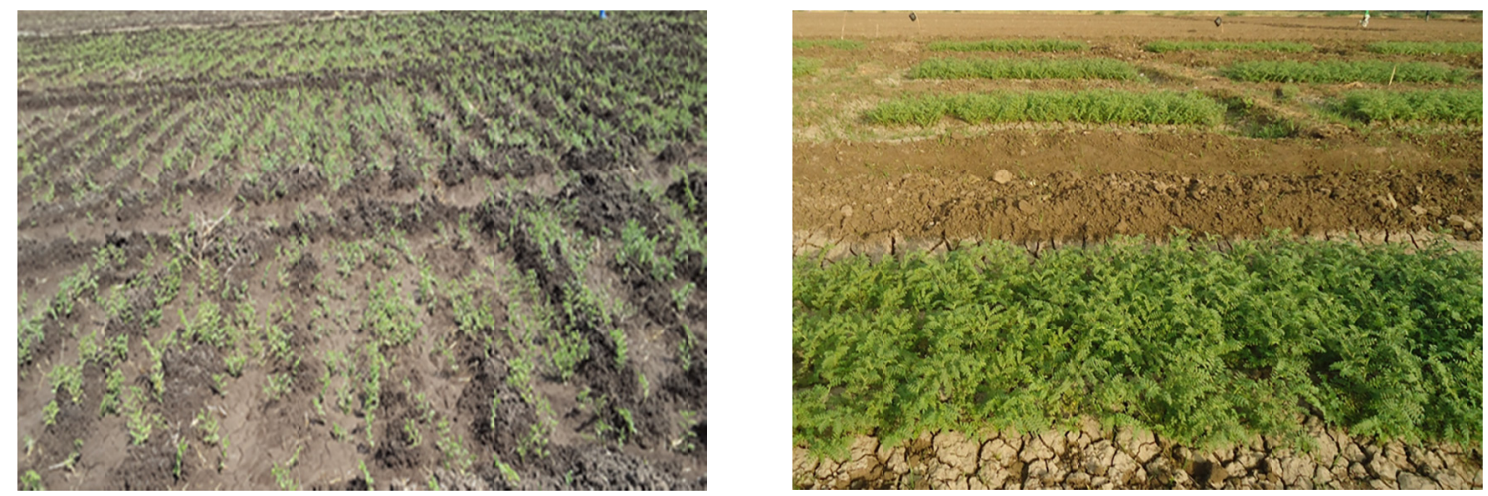

Figure 1. A pictorial view of the field layout to evaluate chickpea genotypes for adaptation to irrigation-based production

\section{Result and Discussion}

All the genotypes under treatment were produced and harvested. There was a significant difference $(P>0.05)$ among the genotypes in their date of maturity. Dates of maturity of the genotypes differed at different locations, possibly due to irrigation management and thermal level. Though all the genotypes were early-maturing, according to the result of combined analysis, only four genotypes (X96TH-52-14/2000 $=106.7 \mathrm{DAS}$, FLIP 02-39C $=107 \mathrm{DAS}, \mathrm{X} 98 \mathrm{TH}-51-1-3=107.9 \mathrm{DAS}$ and ICCV-07313 $=107 \mathrm{DAS}$ ) matured earlier than the others, including the check, in the entire trial (Table 2). Three genotypes at Debre Zeit, one genotype at Ambo and five genotypes at Werer were found maturing earlier than others at their specific locations (Table 2). Similarly, the delay in days to 50\% flowering causes delay in maturity. Bakhsh et al. (2007) and Kanouni (2001) have reported delay in flowering due to irrigation. In conclusion irrigation during any phase of development significantly increases the time required for flowering, podding and physiological and harvest maturity.

Table 2. Date of maturity of Kabuli early under irrigation at different locations (Debre Zeit, Ambo and Werer)

\begin{tabular}{llllll}
\hline \multirow{2}{*}{ No. } & \multirow{2}{*}{ Treatment/Variety } & \multicolumn{3}{c}{ Date of Maturity (days) } \\
\cline { 3 - 5 } & & Debre Zeit & Ambo & Werer & Means \\
\hline 1 & FLIP 02-13C & $120 \mathrm{a}$ & $126 \mathrm{ab}$ & $116 \mathrm{ab}$ & $120.7 \mathrm{abc}$ \\
2 & FLIP 01-2C & $118 \mathrm{a}$ & $128 \mathrm{ab}$ & $120 \mathrm{ab}$ & $122 \mathrm{a}$ \\
3 & FLIP 01-3C & $118 \mathrm{a}$ & $135 \mathrm{a}$ & $121.7 \mathrm{ab}$ & $125 \mathrm{a}$ \\
4 & FLIP 03-59C & $116 \mathrm{ab}$ & $135 \mathrm{a}$ & $107 \mathrm{bc}$ & $119 \mathrm{abcd}$ \\
5 & ICCV-05309 & $115 \mathrm{ab}$ & $128 \mathrm{ab}$ & $99 \mathrm{~cd}$ & $114 \mathrm{bcde}$ \\
6 & FLIP 01-56C & $115 \mathrm{ab}$ & $126 \mathrm{ab}$ & $122 \mathrm{a}$ & $120.9 \mathrm{ab}$ \\
7 & X96TH-52-14/2000 & $114.7 \mathrm{ab}$ & $121 \mathrm{ab}$ & $95 \mathrm{~cd}$ & $106.7 \mathrm{e}$ \\
8 & FLIP 02-39C & $114 \mathrm{ab}$ & $120 \mathrm{ab}$ & $91.7 \mathrm{~d}$ & $107 \mathrm{e}$ \\
9 & ICCV-04305 & $110 \mathrm{bc}$ & $135 \mathrm{a}$ & $95 \mathrm{~cd}$ & $113 \mathrm{cde}$ \\
10 & X98TH-51-1-3 & $110 \mathrm{bc}$ & $114 \mathrm{~b}$ & $100.7 \mathrm{~cd}$ & $107.9 \mathrm{e}$ \\
11 & ICCV-07313 & $109.7 \mathrm{bc}$ & $121 \mathrm{ab}$ & $95 \mathrm{~cd}$ & $107 \mathrm{e}$ \\
12 & Chefe & $109 \mathrm{bc}$ & $128 \mathrm{ab}$ & $95.7 \mathrm{~cd}$ & $112.9 \mathrm{de}$ \\
13 & Ejere & $105 \mathrm{c}$ & $127 \mathrm{ab}$ & $95 \mathrm{~cd}$ & $110.8 \mathrm{e}$ \\
14 & DZ-10-4 & $104 \mathrm{c}$ & $128 \mathrm{ab}$ & $92 \mathrm{~d}$ & $111 \mathrm{e}$ \\
& Mean & 112.8 & 126.6 & 103 & 114 \\
& CV & 4 & 16.8 & 8 & 7 \\
& LSD at 5\% & 7.9 & 14.7 & 3.5 \\
\hline
\end{tabular}

Note. Means followed by different letters in the same column differ from each other by the F-Test $(\mathrm{P}<0.05)$.

In line with these results, the long standing problem of population stand establishment has remained one of the key challenges in irrigated chickpea. In a controlled on-station trial with 3 factors at 2 levels arranged in 8 treatments, i.e., two varieties $\times$ seed priming and non-priming $\times$ pre-watered and post-watered plot; it was 
observed that seed priming in pre-watered plot gave the highest germination (98-100\%) compared to post-watered which gave $75-85 \%$ (Table 3). Concurrently, it was also observed that phenological responses of Kabuli primed seed on pre-watered plot flowered earlier than other setups. This preliminary observation requires further experimentation and validation before substantive publication in a future issue of the journal.

Table 3. Effects of seed priming on two chickpea varieties under different irrigation schemes

\begin{tabular}{|c|c|c|c|c|c|c|c|c|c|c|c|c|c|c|}
\hline \multirow{3}{*}{ Variety } & \multicolumn{6}{|c|}{ Pre-watered plot before planting } & \multicolumn{6}{|c|}{ Post-watered plot after planting } & \multirow{2}{*}{\multicolumn{2}{|c|}{ Phenology }} \\
\hline & \multicolumn{3}{|c|}{ SPT } & \multicolumn{3}{|c|}{ NPT } & \multicolumn{3}{|c|}{ SPT } & \multicolumn{3}{|c|}{ NPT } & & \\
\hline & GP (\%) & DF & SW (1-9) & GP \% & $\mathrm{DF}$ & SW (1-9) & GP (\%) & $\mathrm{DF}$ & SW (1-9) & GP \% & $\mathrm{DF}$ & SW (1-9) & $\mathrm{DF}$ & DM \\
\hline Dimtu & 83 & 41 & 5 & 100 & 43 & 5 & 90 & 43 & 5 & 55 & 40 & 5 & 47 & 110 \\
\hline Habru & 99 & 44 & 3 & 100 & 48 & 3 & 55 & 48 & 1 & 30 & 49 & 3 & 50 & 111 \\
\hline
\end{tabular}

Note. $\mathrm{SPT}=$ Seed priming treatment, $\mathrm{NPT}=$ Non-priming treatment, $\mathrm{GP}=$ Germination percentage, $\mathrm{DF}=$ Days to flowering, $\mathrm{SW}=$ Seedling wilt $\mathrm{DM}=$ Days to maturity.

There was also a substantial difference between the genotypes for 100-seed weight at 0.05 levels. The 100 -seed weight of the genotypes varied across locations; the maximum 100-seed weight for FLIP 02-13C was $45 \mathrm{~g}$ followed by FLIP 01-2C (41.5 g) and FLIP 01-3C (39 g) at Debre Zeit, although the 100-seed weight of these materials at Ambo and Werer were decreasing by an average of $10 \mathrm{~g}$ (Table 4).

The 100-seed weight of only two genotypes (ICCV-07313 $=42.5 \mathrm{~g}$ and ICCV-04305 $=37.8 \mathrm{~g}$ ) were found superior across all locations. Otherwise, 100-seed weight of three genotypes at Debre Zeit, two genotypes at Ambo and three genotypes at Werer were found higher than other genotypes at their respective locations. Similarly, two irrigations at branching and pod formation stages were shown better seeds pod-1 and 1000 -seed weight, as reported by Ray et al. (2011).

Table 4. Mean 100-seed weight of Kabuli early under irrigation at different locations

\begin{tabular}{llllll}
\hline \multirow{2}{*}{ No. } & \multirow{2}{*}{ Treatment/Variety } & \multicolumn{3}{c}{ 100-seed weight } & \\
\cline { 3 - 6 } & & Debre Zeit & Ambo & Werer & Location means \\
\hline 1 & FLIP 02-13C & $45 \mathrm{a}$ & $30.5 \mathrm{c}$ & $29.8 \mathrm{~d}$ & $31 \mathrm{c}$ \\
2 & FLIP 01-2C & $41.5 \mathrm{~b}$ & $31.9 \mathrm{bc}$ & $30 \mathrm{~d}$ & $32 \mathrm{c}$ \\
3 & FLIP 01-3C & $39 \mathrm{bc}$ & $31.9 \mathrm{bc}$ & $28 \mathrm{~d}$ & $31 \mathrm{c}$ \\
4 & FLIP 03-59C & $37.5 \mathrm{~cd}$ & $31.6 \mathrm{bc}$ & $28.6 \mathrm{~d}$ & $31.8 \mathrm{c}$ \\
5 & ICCV-05309 & $35 \mathrm{de}$ & $29 \mathrm{c}$ & $24 \mathrm{e}$ & $28 \mathrm{~d}$ \\
6 & FLIP 01-56C & $34 \mathrm{e}$ & $32.7 \mathrm{bc}$ & $29.8 \mathrm{~d}$ & $33 \mathrm{c}$ \\
7 & X96TH-52-14/2000 & $34 \mathrm{e}$ & $22 \mathrm{~d}$ & $27.6 \mathrm{~d}$ & $26.5 \mathrm{~d}$ \\
8 & FLIP 02-39C & $33 \mathrm{ef}$ & $31.9 \mathrm{bc}$ & $28.7 \mathrm{~d}$ & $31 \mathrm{c}$ \\
9 & ICCV-04305 & $33 \mathrm{ef}$ & $37.7 \mathrm{ab}$ & $34 \mathrm{~b}$ & $37.8 \mathrm{~b}$ \\
10 & X98TH-51-1-3 & $33 \mathrm{ef}$ & $28 \mathrm{~cd}$ & $22.6 \mathrm{e}$ & $26 \mathrm{~d}$ \\
11 & ICCV-07313 & $31.4 \mathrm{gf}$ & $42 \mathrm{a}$ & $40 \mathrm{a}$ & $42.5 \mathrm{a}$ \\
12 & Chefe & $29.5 \mathrm{gh}$ & $32.8 \mathrm{bc}$ & $30.3 \mathrm{~cd}$ & $32 \mathrm{c}$ \\
13 & Ejere & $27.8 \mathrm{~h}$ & $37.7 \mathrm{ab}$ & $32.9 \mathrm{bc}$ & $36.6 \mathrm{~b}$ \\
14 & DZ-10-4 & $12 \mathrm{i}$ & $11 \mathrm{e}$ & $10.9 \mathrm{f}$ & $11 \mathrm{e}$ \\
\hline & Mean & 33 & 30.9 & 28 & 30.9 \\
& CV & 4 & 6 & 5.7 & 8 \\
\hline
\end{tabular}

Commonly, FLIP 02-13C genotypes had demonstrated higher yield than the released variety used as a standard check (Ejere, Chefe and Dz-10-4) at Debre Zeit (Tables 5 and 6). However, the result of combined analysis showed that only one genotype (ICCV-05309 $=3228.8 \mathrm{~kg} / \mathrm{ha}$ or 32 quintals $/$ ha) could out yield the other genotypes over all locations combined. Based on the current available genotypes assessment, we are convinced, from the very good yield performances of X96TH-52-14/2000 $=2489 \mathrm{~kg} / \mathrm{ha}$, FLIP $02-13 \mathrm{C}=2261.8 \mathrm{~kg} / \mathrm{ha}$, FLIP 
$03-59 \mathrm{C}=2222.5 \mathrm{~kg} / \mathrm{ha}, \mathrm{ICCV}-04305=2213.5 \mathrm{~kg} / \mathrm{ha}$, and FLIP $02-39 \mathrm{C}=2094.5 \mathrm{~kg} / \mathrm{ha}$, that irrigation is great avenue for research and development in chickpea production in Ethiopia. Similar to this study, increase in grain yield of chickpea under irrigation has been reported by many authors (Anwar et al., 2003; Pacucci et al., 2006; Bakhsh et al., 2007; Kang et al., 2008; Vinayak et al., 2012). The better performance of certain genotypes after irrigation, as compared to main season, reaffirms the decision to embark upon irrigation systems at scale.

Table 5. Comparison of mean date of maturity (DM), 100-seed weight (HSW) and yield of Kabuli chickpea early under rainfall and irrigation conditions at Debre Zeit

\begin{tabular}{|c|c|c|c|c|c|c|c|}
\hline \multirow[b]{2}{*}{ Genotypes } & \multicolumn{3}{|c|}{ Variables (Irrigation) } & \multicolumn{3}{|c|}{ Variables (Rainfall) } & \multirow[b]{2}{*}{ Genotypes } \\
\hline & $\mathrm{DM}$ & $\begin{array}{l}\text { HSW } \\
(\mathrm{g})\end{array}$ & $\begin{array}{l}\text { Yield } \\
\text { (kg/ha) }\end{array}$ & $\begin{array}{l}\text { Yield } \\
(\mathrm{kg} / \mathrm{ha})\end{array}$ & $\begin{array}{l}\text { HSW } \\
(\mathrm{g})\end{array}$ & $\mathrm{DM}$ & \\
\hline FLIP 02-13C & $120 \mathrm{a}$ & $45 \mathrm{a}$ & $4133 a$ & $2300.5 b$ & 32def & $124 \mathrm{ab}$ & FLIP 02-13C \\
\hline FLIP 01-2C & $118 \mathrm{a}$ & $41.5 b$ & $3600 \mathrm{ab}$ & $2848.6 \mathrm{a}$ & $34.8 \mathrm{~cd}$ & $123 \mathrm{ab}$ & FLIP 01-2C \\
\hline FLIP 01-3C & $118 \mathrm{a}$ & $39 b c$ & $3291 b c$ & $2562.5 \mathrm{ab}$ & $33 \mathrm{de}$ & $123 \mathrm{ab}$ & FLIP 01-3C \\
\hline FLIP 03-59C & $116 a b$ & $37.5 \mathrm{~cd}$ & $3204 \mathrm{bcd}$ & $2964.8 \mathrm{a}$ & $33.9 \mathrm{~d}$ & $118.7 \mathrm{~b}$ & FLIP 03-59C \\
\hline ICCV-05309 & $115 \mathrm{ab}$ & $35 \mathrm{de}$ & $3183 \mathrm{bcd}$ & $1038 \mathrm{cde}$ & $28.7 \mathrm{f}$ & $126 a$ & ICCV-05309 \\
\hline FLIP 01-56C & $115 \mathrm{ab}$ & $34 \mathrm{e}$ & $3169 \mathrm{bcd}$ & $2989 a$ & $33.8 \mathrm{~d}$ & $123.7 \mathrm{ab}$ & FLIP 01-56C \\
\hline Х96ТH-52-14/2000 & $114.7 \mathrm{ab}$ & $34 \mathrm{e}$ & $2983.8 \mathrm{bcd}$ & $1536 \mathrm{c}$ & $29.8 \mathrm{ef}$ & $112 \mathrm{~d}$ & X96TH-52-14/2000 \\
\hline FLIP 02-39C & $114 \mathrm{ab}$ & $33 \mathrm{ef}$ & 2973.6bcd & $1351.9 \mathrm{~cd}$ & $31.9 \mathrm{def}$ & $122.7 \mathrm{ab}$ & FLIP 02-39C \\
\hline ICCV-04305 & $110 \mathrm{bc}$ & $33 \mathrm{ef}$ & $2968.5 \mathrm{bcd}$ & $1080.6 \mathrm{cde}$ & $40 \mathrm{~b}$ & $122 \mathrm{ab}$ & ICCV-04305 \\
\hline Х98ТН-51-1-3 & $110 \mathrm{bc}$ & $33 \mathrm{ef}$ & $2781.9 \mathrm{~cd}$ & 1005.6de & $30 \mathrm{ef}$ & $108 \mathrm{~d}$ & Х98ТH-51-1-3 \\
\hline ICCV-07313 & $109.7 \mathrm{bc}$ & $31.4 \mathrm{gf}$ & $2615.7 \mathrm{cde}$ & $730.6 \mathrm{ef}$ & $49 a$ & $112.3 \mathrm{~cd}$ & ICCV-07313 \\
\hline Chefe & $109 b c$ & $29.5 \mathrm{gh}$ & $2453.7 \mathrm{de}$ & $2256 b$ & $32.5 \mathrm{de}$ & $111 d$ & Chefe \\
\hline Ejere & $105 c$ & $27.8 \mathrm{~h}$ & $1946 \mathrm{e}$ & $1543 c$ & $38 \mathrm{bc}$ & $111 d$ & Ejere \\
\hline DZ-10-4 & $104 \mathrm{c}$ & $12 \mathrm{i}$ & $1883 \mathrm{e}$ & $352 \mathrm{f}$ & $10.8 \mathrm{~g}$ & $118 \mathrm{bc}$ & DZ-10-4 \\
\hline Mean & 112.8 & 33 & 2942 & 1754 & 32.8 & 118.4 & Mean \\
\hline $\mathrm{CV}$ & 4 & 4 & 15.9 & 17 & 6.5 & 3 & $\mathrm{CV}$ \\
\hline LSD at $5 \%$ & 7.9 & 2.6 & 789 & 520 & 3.5 & 6 & LSD at $5 \%$ \\
\hline
\end{tabular}

Table 6. Mean yield of Kabuli early under irrigation over location

\begin{tabular}{llllll}
\hline \multirow{2}{*}{ No. } & \multirow{2}{*}{ Treatment/Variety } & \multicolumn{4}{c}{ Mean yield $\left(\mathrm{kg} \mathrm{ha}^{-1}\right)$} \\
\cline { 3 - 6 } & & Debre Zeit & Ambo & Werer & Location Means \\
\hline 1 & CFLIP 02-13C & $4133 \mathrm{a}$ & $3254 \mathrm{~cd}$ & $557.6 \mathrm{ef}$ & $2261.8 \mathrm{bcde}$ \\
2 & FLIP 01-2C & $3600 \mathrm{ab}$ & $2590.8 \mathrm{~cd}$ & $626.5 \mathrm{e}$ & $1890 \mathrm{f}$ \\
3 & FLIP 01-3C & $329 \mathrm{bc}$ & $2820.7 \mathrm{c}$ & $667 \mathrm{e}$ & $2152 \mathrm{cdef}$ \\
4 & FLIP 03-59C & $3204 \mathrm{bcd}$ & $3928.9 \mathrm{ab}$ & $855 \mathrm{~d}$ & $2222.5 \mathrm{bcdef}$ \\
5 & ICCV-05309 & $3183 \mathrm{bcd}$ & $4315.9 \mathrm{a}$ & $1237 \mathrm{bc}$ & $3228.8 \mathrm{a}$ \\
6 & FLIP 01-56C & $3169 \mathrm{bcd}$ & $2684 \mathrm{~cd}$ & $454.7 \mathrm{f}$ & $2040.9 \mathrm{def}$ \\
7 & X96TH-52-14/2000 & $2983.8 \mathrm{bcd}$ & $2924 \mathrm{c}$ & $1339 \mathrm{ab}$ & $2489 \mathrm{cc}$ \\
8 & FLIP 02-39C & $2973.6 \mathrm{bcd}$ & 2689.7 & $977.9 \mathrm{~d}$ & $2094.5 \mathrm{def}$ \\
9 & ICCV-04305 & $2968.5 \mathrm{bcd}$ & $1655 \mathrm{e}$ & $1385 \mathrm{a}$ & $2213.5 \mathrm{bcdef}$ \\
10 & X98TH-51-1-3 & $2781.9 \mathrm{~cd}$ & $2074.8 \mathrm{de}$ & $913.6 \mathrm{~d}$ & $1923 \mathrm{ef}$ \\
11 & ICCV-07313 & $2615.7 \mathrm{cde}$ & $716.5 \mathrm{f}$ & $1315 \mathrm{ab}$ & $1325.9 \mathrm{~g}$ \\
12 & Chefe & $2453.7 \mathrm{de}$ & $3233 \mathrm{bc}$ & $1220 \mathrm{bc}$ & $2540.9 \mathrm{~b}$ \\
13 & Ejere & $1946 \mathrm{e}$ & $2729.9 \mathrm{~cd}$ & $1158 \mathrm{c}$ & $2357 \mathrm{bcd}$ \\
14 & DZ-10-4 & $1883 \mathrm{e}$ & $2886.5 \mathrm{c}$ & $958.6 \mathrm{~d}$ & $2378.9 \mathrm{bcd}$ \\
& Mean & 2942 & 2750 & 976 & 2222.85 \\
& CV & 15.9 & 15 & 8.7 & 17 \\
& LSD at 5\% & 789 & 709.9 & 142.7 & 168.7 \\
\hline
\end{tabular}


In case of desi types, all of the genotypes reached their maturity date in under four months. The 100 -seed weight of six genotypes (ICCX-090013-F2-P177-BP $=35 \mathrm{~g}$, ICCX-090013-F2-P216-BP $=34.8 \mathrm{~g}$, ICCX-090013-F2$\mathrm{P} 276-\mathrm{BP}=34.7 \mathrm{~g}$, ICCX-090013-F2-P248-BP $=33 \mathrm{~g}$, ICCX-090013-F2-P107-BP = 32 gm and ICCX-090013$\mathrm{F} 2-\mathrm{P} 173-\mathrm{BP}=32 \mathrm{~g}$ ) were found higher than the other, and also the yield of eight genotypes (ICCX-090013-F2P216-BP, ICCX-090013-F2-P129-BP, ICCX-090013-F2-P103-BP, ICCX-090013-F2-P215-BP, ICCX-090013F2-P276-BP, ICCX-090013-F2-P163-BP, ICCX-090013-F2-P173-BP and ICCX-090013-F2-P265-BP) were found promising with respect to high yields $(>20 \mathrm{~kg} / \mathrm{ha}$ ) out of the genotypes under irrigation (Table 7). The same result was reported by Mansur et al. (2010), and Dogan et al. (2012). According to Fardin et al. (2013) growth continued during all growth stages, but for best growth of chickpea and for higher yields, irrigation is better. The influence of disease and insects was minimal during the trial season; this could be an opportunity for studying the limitation of stress levels and their effect on yield and product quality. Because of the new cropping system, the climatic and environmental conditions may not support development of pests, as in the case of the main cropping seasons.

Table 7. Mean date of maturity (DM), 100-seed weight (HSW) and yield of desi chickpea under irrigation over location (The materials were obtained from ICRISAT)

\begin{tabular}{|c|c|c|c|c|}
\hline \multirow{2}{*}{ No. } & \multirow{2}{*}{ Treatment/Variety } & \multicolumn{3}{|c|}{ Variables } \\
\hline & & $\mathrm{DM}$ & HSW (g) & Yield (kg/ha) \\
\hline 1 & ICCX-090013-F2-P177-BP & $101.7 \mathrm{abc}$ & $35.6 \mathrm{a}$ & 2274.5defghij \\
\hline 2 & ICCX-090013-F2-P163-BP & $102 \mathrm{abc}$ & 29 fghi & $2475.5 b c d e f g h$ \\
\hline 3 & ICCX-090013-F2-P276-BP & $101 \mathrm{abc}$ & $34.7 \mathrm{bc}$ & $2466.7 \mathrm{bcdefgh}$ \\
\hline 4 & ICCX-090013-F2-P234-BP & $100 \mathrm{abc}$ & 30efghi & 1936hij \\
\hline 5 & ICCX-090013-F2-P120-BP & $93 \mathrm{c}$ & $23.71 \mathrm{~m}$ & 2422.7cdefghi \\
\hline 6 & ICCX-090013-F2-P107-BP & $96.7 \mathrm{abc}$ & $32 \mathrm{bcde}$ & 2053.7fghij \\
\hline 7 & ICCX-090013-F2-P215-BP & $107 \mathrm{ab}$ & 30.5defghi & 2748abcde \\
\hline 8 & ICCX-090013-F2-P108-BP & $104 \mathrm{abc}$ & $32 \mathrm{bcde}$ & $1805.6 \mathrm{ij}$ \\
\hline 9 & ICCX-090013-F2-P147-BP & $103 \mathrm{abc}$ & $30.5 \mathrm{defgh}$ & 2305defghi \\
\hline 10 & ICCX-090013-F2-P103-BP & $101 \mathrm{abc}$ & $231 \mathrm{~m}$ & $2874.5 \mathrm{abcd}$ \\
\hline 11 & ICCX-090013-F2-P245-BP & $97 \mathrm{abc}$ & $25 \mathrm{jkl}$ & 2420.8 cdefghi \\
\hline 12 & ICCX-090013-F2-P129-BP & $108.7 \mathrm{a}$ & 27.7hijk & $2954 a b c$ \\
\hline 13 & ICCX-090013-F2-P216-BP & $98 \mathrm{abc}$ & $34.8 \mathrm{bc}$ & $3283.8 \mathrm{a}$ \\
\hline 14 & ICC-4958 & $98 \mathrm{abc}$ & 30defghi & 2306defghi \\
\hline 15 & ICCX-090013-F2-P145-BP & $104 \mathrm{abc}$ & 31.6def & 2225 efghij \\
\hline 16 & ICCX-090013-F2-P145-BP & $102 \mathrm{abc}$ & $27.6 \mathrm{ijk}$ & 2363.9cdefghi \\
\hline 17 & ICCX-090013-F2-P173-BP & $96.7 \mathrm{abc}$ & $32 \mathrm{bcde}$ & 2738.9abcde \\
\hline 18 & ICCX-090013-F2-P284-BP & $94 b c$ & $25 \mathrm{kl}$ & $1659 \mathrm{j}$ \\
\hline 19 & JL-11 & $105.7 \mathrm{abc}$ & 30.9defg & 2591.7bcdefg \\
\hline 20 & ICCX-090013-F2-P105-BP & $96.7 \mathrm{abc}$ & $22 \mathrm{~m}$ & 2576bcdefg \\
\hline 21 & ICCX-090013-F2-P175-BP & $98 \mathrm{abc}$ & $23.61 \mathrm{~m}$ & 1969ghij \\
\hline 22 & ICCX-090013-F2-P265-BP & $98 \mathrm{abc}$ & $32 \mathrm{cde}$ & 2661abcdef \\
\hline 23 & ICCX-090013-F2-P248-BP & $97 \mathrm{abc}$ & $33 \mathrm{bcd}$ & $1668.5 \mathrm{j}$ \\
\hline \multirow[t]{4}{*}{24} & ICCX-090013-F2-P223-BP & $98 \mathrm{abc}$ & 28ghij & 2348cdefghi \\
\hline & Mean & 100.6 & 29.8 & 2407.5 \\
\hline & $\mathrm{CV}$ & 8 & 6 & 15.9 \\
\hline & LSD at $5 \%$ & 13.5 & 2.9 & 631 \\
\hline
\end{tabular}

\section{Conclusion and Recommendation}

The irrigation trial undertaken on promising chickpea genotypes in 2012/13 demonstrated that irrigation is one of the major contributors in chickpea production system. There is untapped potential in production, productivity and quality to be exploited using the irrigation system. All the genotypes sown from Kabuli and desi varieties set quality seed at all locations. Except for termites at Werer, and wilt on one genotype at Debre Zeit, the occurrence of disease and insects was not detected. At some locations, the yield of some genotypes stood out as more important for our irrigation trial. Although the genotypes, especially the Kabuli types, were introduced for other purposes, they were also found to be adaptable to irrigation-based production. Of these, some genotypes 
performed better even in main season at Debre Zeit, as evaluated by all parameters used for analysis. From the results, we recommend that, even though all materials were not found equally important for all parameters evaluated, those genotypes which best demonstrated yield, seed size and maturity be included in the breeding program. So, genotypes with high 100-seed weight are used as parents for crossing with genotypes with low 100 -seed weight, but giving high yields. It was also interesting to deal with optimization of irrigation frequency, developing responsive and suitable cultivars for the irrigation system and developing $\mathrm{G} \times \mathrm{E}$ stable commercial varieties in the research design. In conclusion, ICCX-090013-F2-P120-BP, FLIP 02-39C, X96TH-52-14/2000, X98TH-51-1-3 and ICCV-07313 for maturity; ICCX-090013-F2-P177-BP, FLIP 02-13C and ICCV-07313 for 100-seed weight; and ICCX-090013-F2-P216-BP, FLIP 02-13C and ICCV-05309 for yield were identified for further breeding work and yield trial for release for irrigation-based production areas.

\section{Reference}

Anwar, M. R., McKenzie, B. A., \& Hill, G. D. (2003). Water-use efficiency and the effect of water deficits on crop growth and yield of Kabuli chickpea in a cool-temperate sub humid climate. J. Agric. Sci., 141, 285-301. https://doi.org/10.1017/S0021859603003630

Brick, H., Knewtson, S., \& Grusak, M. A. (2003). Chickpea leaves as vegetable green for humans: Evolution of mineral composition. J. Sci. Food Agric., 83, 945-950. https://doi.org/10.1002/jsfa.1427

Central Statistical Authority (CSA). (2015). Agricultural sample survey, Report on, area and production for major crops (private peasant holdings, meher season). Addis Ababa, Ethiopia.

Central Statistical Authority (CSA). (2016). Agricultural sample survey, Report on, area and production for major crops (private peasant holdings, meher season). Addis Ababa, Ethiopia.

Dogan, E., Kahraman, A., Bucak, B., Kirnak, H., \& Guldur, M. E. (2012). Varying irrigation rates effect on yield and yield components of chickpea. Irrig Sci, 31, 903-909. https://doi.org/10.1007/s00271-012-0367-8

Fardin, M., Mokhtar, G., Saeid, J.-H., \& Parviz, S. (2013). Effect of Supplementary Irrigation on growth analysis of Chickpea (Cicer arietinum L.). Intl J Agri Crop Sci., 5(14), 1595-1600.

Gaur, P. M., Jukanti, A. K., \& Varshney, R. K. (2012). Impact of Genomic Technologies on Chickpea Breeding Strategies. Grain Legumes Research Program, International Crops Research Institute for the Semi-Arid Tropics (ICRISAT), Patancheru, Andhra Pradesh, India. https://doi.org/10.3390/agronomy2030199

Hulse, J. H. (1991). Nature, composition and utilization of grain legumes (pp. 11-27). Uses of tropical Legumes: Proceedings of a Consultants' Meeting, 27-30 March 1989, ICRISAT Center. ICRISAT, Patancheru, A.P., India.

Jukanti, A. K., Gaur, P. M., Gowda, C. L. L., \& Chibbar, R. N. (2012). Nutritional quality and health benefits of chickpea (Cicer arietinum L.): A review. Journal of Nutrition, 108, S11-S26. https://doi.org/10.1017/ S0007114512000797

Kang, S., McKenzie, B. A., \& Hill, G. D. (2008). .Effect of irrigation on growth and yield of Kabuli chickpea (Cicer arietinum L.) and narrow-leafed lupin (Lupinus angustifolius L.). Agronomy New Zealand, 38. Agricultural Science Department, Faculty of Agriculture and Life Sciences, Lincoln University, Lincoln, New Zealand

Kanouni, H. (2001). The yielding ability and adaptability of chickpea cultivars under rain fed conditions of Kurdistan. Seed Pl., 17(1).

Kumar, N. (2016). Food Seed Health of Chick Pea (Cicer arietinum L.) at Panchgaon, Gurgaon, India. Adv Crop Sci Tech, 4, 229. https://doi.org/10.4172/23298863.1000229

Mansur, C. P., Palled, Y. B., Salimath, P. M., \& Halikatti, S. I. (2010). An analysis of dry matter production, growth and yield in Kabuli chickpea as influenced by dates of sowing and irrigation levels. Karnataka J. Agric. Sci., 23(3), 457-460.

Muehlbauer, F. J., Short, R. W., \& Kaiser, W. J. (1982). Description and culture of garbanzo beans. Coop. Ext. Publ. EB 1112, Washington State Univ., Pullman.

Pacucci, G., Troccoli, C., \& Leoni, B. (2006). Supplementary Irrigation on Yield of Chickpea Genotypes in a Mediterranean Climate. Agricultural Engineering International: The CIGR Ejournal, VIII (1), Manuscript LW 04005. 
Ray, M, Nanda, M. K., \& Khan, D. K. (2011). Effect of date of sowing and irrigation on seed yield, yield attributes and water use of Chickpea (Cicer arietinum L.) at lower Gangetic plains of West Bengal. Journal of Crop and Weed, 7(2), 30-32.

Vinayak, N., Halepyati, A. S., \& Koppalkar, B. G. (2012). Growth and yield of late sown chickpea as influenced by irrigation methods, genotypes and planting densities. Karnataka J. Agric. Sci., 25(2), 267-269.

\section{Copyrights}

Copyright for this article is retained by the author(s), with first publication rights granted to the journal.

This is an open-access article distributed under the terms and conditions of the Creative Commons Attribution license (http://creativecommons.org/licenses/by/4.0/). 